\title{
CARACTERIZAÇÃO DE CINZA OBTIDA POR COMBUSTÃo DE CASCA DE ARROZ EM REATOR DE LEITO FLUIDIZADO
}

\author{
Juan Daniel Martínez Angel e Tatiana Gisset Pineda Vásquez \\ Grupo de Investigaciones Ambientales, Universidad Pontifícia Bolivariana, Medellín, Colômbia \\ Janaina Accordi Junkes e Dachamir Hotza* \\ Departamento de Engenharia Química e Engenharia de Alimentos, Universidade Federal de Santa Catarina, 88040-900 Florianópolis \\ - SC, Brasil \\ Recebido em 24/1/08; aceito em 4/12/08; publicado na web em 15/4/09
}

\begin{abstract}
CHARACTERIZATION OF ASH FROM COMBUSTION OF RICE HUSK IN A FLUIDIZED BED REACTOR. The rice husk combustion in a bubbling and atmospheric fluidized bed reactor was investigated. This paper presents the rice husk ash characterization employing the techniques of X-ray diffraction (XRD), X-ray fluorescence (XRF), and scanning electron microscopy (SEM) among others. After combustion, a rice husk ash containing $93 \%$ amorphous silica and $<3 \%$ unburned char was produced. Methods usually applied to fixed bed considering external sources of energy and high reaction times were employed. Thus, the potential of this type of reactors with respect to speed, continuity and self-sufficiency energy of the process was shown.
\end{abstract}

Keywords: amorphous silica; rice husk; fluidized bed.

\section{INTRODUÇÃO}

Atualmente, devido ao aumento da produção agroindustrial, é crescente a preocupação com o destino dos resíduos de processamento, tais como a casca de arroz (CA). Para um melhor aproveitamento material e energético, permitindo uma produção mais limpa e um desenvolvimento mais sustentável, a CA pode ser usada como combustível renovável na própria unidade de fabricação, por exemplo, em geradores de vapor. A combustão da CA gera gases quentes com baixos teores de contaminantes, como monóxido de carbono e material particulado.

A CA é o revestimento protetor formado durante o crescimento do grão de arroz, composta por quatro camadas estruturais: epiderme externa, coberta com uma espessa cutícula de células silificadas; esclerênquima ou fibra hipoderme, com parede lignificada; célula parênquina esponjosa e epiderme interna. ${ }^{1}$ Os principais componentes da casca são celulose e hemicelulose (50\%), além de lignina (26\%) e componentes orgânicos (4\%), como óleos e proteínas. A quantidade restante inclui os materiais inorgânicos $\left(\mathrm{SiO}_{2}, \mathrm{Al}_{2} \mathrm{O}_{3}, \mathrm{~K}_{2} \mathrm{O}, \mathrm{Na}_{2} \mathrm{O}\right.$, $\left.\mathrm{MgO}, \mathrm{CaO}, \mathrm{Fe}_{2} \mathrm{O}_{3}, \mathrm{MnO}, \mathrm{P}_{2} \mathrm{O}_{5}\right){ }^{1}$

A cinza de casca de arroz (CCA) é o produto da transformação termoquímica da CA. As condições dos diferentes processos de conversão (pirólise, gaseificação e/ou combustão) determinam as características físico-químicas do produto final. A CCA corresponde a 14 a 25\% da CA, dependendo da variedade do arroz, do clima e do solo da região produtora. ${ }^{2-4} \mathrm{O}$ principal componente da CCA gerada a moderadas temperaturas de combustão é a sílica amorfa $\left(\mathrm{SiO}_{2}\right) \mathrm{em}$ composições compreendidas entre 80 e $97 \% .4,5$

$\mathrm{Na}$ natureza a sílica pode ser encontrada pura, hidratada ou na forma de minerais. Também pode ser amorfa ou cristalina, dependendo da organização na estrutura atômica. A sílica cristalina também pode se apresentar em três diferentes formas (quartzo, tridimita ou cristobalita), nas quais os átomos adotam arranjos ordenados e repetitivos, formando estruturas tridimensionais periódicas. Por outro lado, a sílica amorfa é uma estrutura formada de átomos com orientação somente a curta distância, de fácil moagem e altamente

\footnotetext{
*e-mail: dhotza@gmail.com
}

reativa, que pode ser utilizada como matéria-prima nas indústrias cerâmica (pigmentos, abrasivos), de materiais de construção civil ou de produtos eletrônicos, entre outros. ${ }^{6,7}$

A fluidização é uma tecnologia adequada para converter diversos resíduos agrícolas em energia, devido a suas vantagens inerentes de flexibilidade de combustível, baixas temperaturas e condições isotérmicas de operação. ${ }^{8}$ A fluidização é uma operação em que um sólido entra em contato com um líquido ou um gás, de tal forma que o conjunto adquire características similares às de fluidos, de modo que as taxas de transferência de massa e energia são elevadas. ${ }^{9}$

Um reator de leito fluidizado (RLF) consiste de uma câmara de reação que contém partículas suportadas por uma placa distribuidora e mantidas em suspensão por um fluido que as atravessa em sentido ascendente. A tecnologia de leito fluidizado é capaz de produzir cinza amorfa a partir de CA com baixo teor de carbono não queimado em intervalos curtos de reação. ${ }^{10}$

A partir da combustão da biomassa de CA é gerado calor que pode ser utilizado em diversos processos industriais. A temperatura de combustão pode ser controlada com base na relação ar-combustível no reator de leito fluidizado e, por conseqüência, gerar resíduos com características distintas.

O presente trabalho apresenta a caracterização de CCA obtida sob condições variáveis de combustão em leito fluidizado, como velocidade de fluidização e fluxo de CA. As técnicas de caracterização incluem análise química por fluorescência de raios X (FRX), análise cristalográfica por difração de raios X (DRX), distribuição de tamanho de partícula (DTP) por difração a laser e por peneiramento, e análise microestrutural através de microscopia eletrônica de varredura (MEV).

\section{PARTE EXPERIMENTAL}

\section{Material}

A CA foi adquirida na forma prensada em pacotes de $70 \mathrm{~kg}$ (Agucatala, Medellín, Colômbia). A CA não sofreu nenhum processamento prévio antes de ser alimentada no RLF. Na Tabela 1, apresentam-se as análises elementar e imediata da CA utilizada na pesquisa. 
Tabela 1. Análises elementar e imediata da casca de arroz

\begin{tabular}{lcc}
\hline Análise & Constituinte & Teor $(\%)$ \\
\hline \multirow{3}{*}{ Elementar } & Carbono & 36,6 \\
& Hidrogênio & 5,83 \\
& Nitrogênio & 3,31 \\
& Oxigênio & 36,5 \\
Imediata & Enxofre & 0,01 \\
& Outros elementos & 17,61 \\
& Voláteis & 57,7 \\
& Umidade residual & 9,3 \\
\hline
\end{tabular}

\section{Métodos}

Reator de leito fluidizado

O equipamento utilizado para a obtenção da CCA foi um RLF, disponível na empresa Premac S.A., Medellín, Colômbia. Tal equipamento é de propriedade do Grupo de Pesquisas Ambientais, Universidade Pontifícia Bolivariana, Medellín, Colômbia.

As dimensões do RLF são $30 \mathrm{~cm}$ de diâmetro interno e $3 \mathrm{~m}$ de altura, com expansão a $40 \mathrm{~cm}$ na zona do freeboard. O reator tem um silo de armazenamento de material, com um sistema de alimentação composto por duas roscas para dosagem e alimentação. Conta, ainda, com um soprador de ar de alta pressão com instrumentação (medidor térmico diferencial) para a medição da quantidade de fluido que ingressa no reator e um aquecedor de gás natural destinado ao pré-aquecimento do leito.

\section{Combustão em leito fluidizado}

Inicialmente, foram colocados $40 \mathrm{~kg}$ de areia no reator para se obter uma altura de leito estático de $40 \mathrm{~cm}$. Em seguida, procedeuse ao aquecimento até $400{ }^{\circ} \mathrm{C}$. Uma vez atingida esta temperatura, desligou-se o aquecedor e introduziu-se a CA, fixando-se as condições estequiométricas de combustão até ser atingida uma temperatura média de $700{ }^{\circ} \mathrm{C}$ no leito. Posteriormente, controlou-se a descarga de CA pelo ajuste da velocidade de fluidização, para que a temperatura, em nenhum dos pontos de medição ao longo do reator, fosse maior que $700{ }^{\circ} \mathrm{C}$. De acordo com experimentos previamente realizados, os valores de velocidade de fluidização utilizados garantem o desenvolvimento da fluidização. Baixas velocidades de fluidização correspondem a maiores tempos de residência das partículas, que levam a melhores condições para a transformação do carbono da casca. ${ }^{15}$ Por outro lado, velocidades de fluidização maiores favorecem melhores condições de mistura, assim como a diminuição da segregação de partículas. ${ }^{11-13}$

Temperaturas superiores a $700{ }^{\circ} \mathrm{C}$ promovem a transformação de sílica amorfa para sílica cristalina. ${ }^{6,10}$ Após ser atingido o equilíbrio termodinâmico, as cinzas produzidas no reator foram coletadas em um tambor hermético e recolhidas para análise.

Alguns pesquisadores reportam temperaturas de transição de sílica amorfa a cristalina entre 700 e $900{ }^{\circ} \mathrm{C} . .^{4,8,10}$ Uma possível explicação pode estar no potássio contido na casca de arroz, atribuído às condições geográficas, variações climáticas, composições químicas do solo e uso de fertilizantes na colheita do grão. $\mathrm{O}$ potássio contido na CA acelera tanto a fusão das partículas, pela formação de eutéticos, quanto a cristalização das diferentes formas da sílica. ${ }^{4,8,10}$ Além disso, grandes quantidades de potássio e outros sais alcalinos têm efeito desfavorável na obtenção de sílica amorfa, causando a formação de aglomerados no interior do reator, que podem levar à desfluidização do leito. ${ }^{14}$ Por outro lado, o conteúdo de carbono que ainda não foi transformado pode ficar aprisionado na cinza, dificultando sua conversão inclusive a temperaturas elevadas. ${ }^{2,8}$

$\mathrm{Na}$ Tabela 2, são apresentadas as velocidades de fluidização em condições normais de pressão e temperatura (1 atm e 273,15 K) e os fluxos de CA utilizados nos experimentos.

Tabela 2. Condições de operação do reator de leito fluidizado

\begin{tabular}{lcc}
\hline Teste & $\begin{array}{c}\text { Velocidade normal } \\
\text { de fluidização } \\
(\mathrm{m} / \mathrm{s})\end{array}$ & Alimentação de CA $(\mathrm{kg} / \mathrm{h})$ \\
\hline A & 0,134 & 5,67 \\
B & 0,143 & 4,53 \\
C & 0,139 & 7,16 \\
D & 0,149 & \\
\hline
\end{tabular}

Caracterização química e física

As análises e os ensaios de caracterização da CCA foram executados na Universidade Nacional da Colômbia (UNC), na empresa Andercol S.A., em Medellín, Colômbia; na Universidade Federal de Santa Catarina, Florianópolis, Brasil, e no Centro de Tecnologia em Materiais (CTCMAT), Criciúma, Brasil.

\section{Difração de raios $X(D R X)$}

$\mathrm{O}$ equipamento utilizado nesta técnica foi um difratômetro PANalytical, modelo $X^{\prime}$ Pertpro-MPD, com radiação K $\alpha$, potência de $45 \mathrm{kV}$ e corrente de $40 \mathrm{~mA}$, disponível na UNC. As análises foram realizadas em amostras na forma de pó, sem nenhum tipo de preparação prévia, no intervalo de medida de $2 \theta$ entre 15 e $70^{\circ}$.

\section{Fluorescência de raios $X(F R X)$}

As análises foram realizadas em um equipamento Philips, modelo PW 2400, com tubo de $3 \mathrm{~kW}$ e alvo de ródio, pertencente ao CTCMAT. Foram usados os procedimentos de operação do equipamento e as normas NBR $9644^{15}$ e NBR $12860^{16}$.

\section{Distribuição de tamanho de partícula (DTP)}

Para a determinação da DTP, foi utilizado um difratômetro a laser Microtrac, modelo S3000, na empresa Andercol S.A.. As amostras foram preparadas em forma de suspensão e submetidas à agitação para completa desaglomeração das partículas.

As amostras também foram classificadas por peneiramento, em um agitador automático, com oito peneiras de malhas 60, 80, 100, 140, 200 e 270 mesh (respectivamente, 250, 177, 149, 105, 74 e 53 $\mu \mathrm{m})$, de acordo com norma ASTM E-11. ${ }^{17}$

\section{Microscopia eletrônica de varredura (MEV)}

$\mathrm{O}$ equipamento utilizado foi um microscópio Philips, modelo XL-30 com filamento de tungstênio, disponível na UFSC. As amostras foram dispostas sobre fita de carbono e, por não possuírem caráter condutor, foram metalizadas com uma fina camada de ouro. 


\section{RESULTADOS E DISCUSSÃO}

\section{Combustão em leito fluidizado}

Na Tabela 3, são apresentadas as temperaturas médias do leito, obtidas a partir das variáveis controladas de entrada, as velocidades de fluidização nas condições reais de temperatura (Tabela 2) e os excessos de ar determinados a partir da alimentação de CA.

Tabela 3. Parâmetros gerais de combustão da CA em leito fluidizado

\begin{tabular}{lccc}
\hline Teste & $\begin{array}{c}\text { Velocidade real } \\
\text { de fluidização } \\
(\mathrm{m} / \mathrm{s})\end{array}$ & $\begin{array}{c}\text { Temperatura } \\
\text { média }\left({ }^{\circ} \mathrm{C}\right)\end{array}$ & $\begin{array}{c}\text { Excesso de ar } \\
(\%)\end{array}$ \\
\hline A & 0,465 & 672 & 68 \\
B & 0,474 & 635 & 120 \\
C & 0,464 & 637 & 99 \\
D & 0,498 & 640 & 91 \\
\hline
\end{tabular}

As condições de operação utilizadas, em particular as quantidades de excesso de ar, determinadas a partir da alimentação de CA, mostram coerência com os valores da temperatura média do leito medidas nos experimentos: quanto maior a quantidade de ar na reação, menor a temperatura, como mostra a Figura 1.

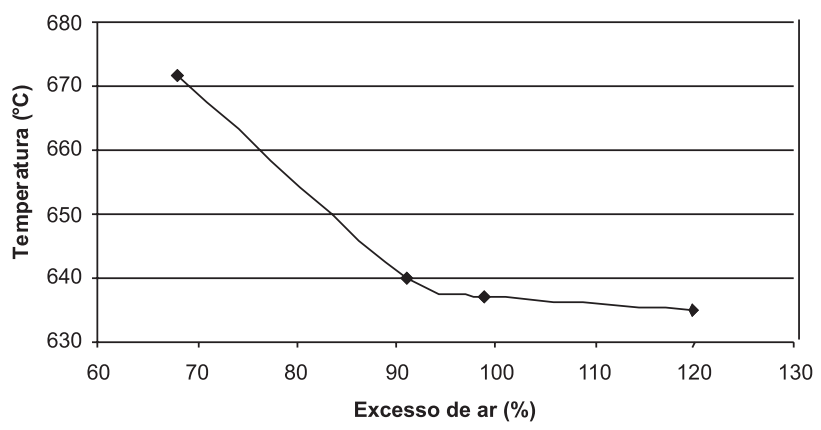

Figura 1. Temperatura do leito fluidizado em função do excesso de ar

\section{Composição química da CCA}

Na Tabela 4 apresenta-se a composição química da CCA para as diferentes condições de velocidade de fluidização e alimentação de CA. Em geral, os resultados da análise química mostraram $\mathrm{SiO}_{2}$ como principal componente, com valores superiores a $93 \%$ em massa, seguido por $\mathrm{K}_{2} \mathrm{O}(<2,5 \%)$ e $\mathrm{P}_{2} \mathrm{O}_{5}(<1 \%)$. De acordo com a análise imediata mostrada na Tabela 1, a sílica presente na cinza corresponde aproximadamente a $16 \%$ de $\mathrm{SiO}_{2}$ na casca.

Experimentos de obtenção de sílica a partir de calcinação de CA em forno elétrico, a $700{ }^{\circ} \mathrm{C}$ durante $4 \mathrm{~h}$, reportaram quantidades de $\mathrm{C}$, $\mathrm{SiO}_{2}$ e $\mathrm{K}_{2} \mathrm{O}$ de 4,2; 90,0 e 2,0\%, respectivamente. ${ }^{2}$ Os pesquisadores ressaltaram o efeito do potássio sobre a fixação do carbono na cinza, em razão da dissociação do $\mathrm{K}_{2} \mathrm{O}$ a $347{ }^{\circ} \mathrm{C}$ e formação do potássio elementar, cujo ponto de fusão é $63,8^{\circ} \mathrm{C}$. No caso de transformações termoquímicas em leito fluidizado, a presença de carbono nas cinzas é atribuída ao tempo de residência da casca no reator, o qual está relacionado à velocidade de fluidização e à altura do reator. ${ }^{8,12}$

Os resultados obtidos não mostram influência apreciável das variáveis de entrada (velocidade de fluidização e alimentação de
Tabela 4. Composição química da CCA em porcentagem mássica

\begin{tabular}{lcccc}
\hline \multirow{2}{*}{ Óxidos } & \multicolumn{4}{c}{ Amostras } \\
\cline { 2 - 5 } & 93,58 & 93,16 & 93,61 & 93,74 \\
\hline $\mathrm{SiO}_{2}$ & 0,13 & 0,16 & 0,14 & 0,10 \\
$\mathrm{Al}_{2} \mathrm{O}_{3}$ & 0,02 & 0,02 & 0,01 & 0,02 \\
$\mathrm{Na}_{2} \mathrm{O}$ & 2,15 & 2,27 & 2,17 & 2,11 \\
$\mathrm{~K}_{2} \mathrm{O}$ & 0,50 & 0,45 & 0,47 & 0,37 \\
$\mathrm{MgO}$ & 0,34 & 0,37 & 0,36 & 0,31 \\
$\mathrm{CaO}$ & 0,10 & 0,07 & 0,09 & 0,09 \\
$\mathrm{Fe}_{2} \mathrm{O}_{3}$ & 0,14 & 0,14 & 0,13 & 0,12 \\
$\mathrm{MnO}$ & 0,62 & 0,67 & 0,53 & 0,50 \\
$\mathrm{P}_{2} \mathrm{O}_{5}$ & 0,02 & 0,02 & 0,02 & 0,01 \\
$\mathrm{TiO}_{2}$ & 2,41 & 2,67 & 2,47 & 2,63 \\
$\mathrm{Perda}$ & 100 & 100 & 100 & 100 \\
Total & & &
\end{tabular}

CA) na composição química da CCA, dentro da faixa de valores pesquisada neste trabalho, em contraste com dados encontrados na literatura. ${ }^{11-14,18}$

Ao se compararem os valores ótimos de velocidade de fluidização e carbono residual encontrados por Rozainee et al. $.{ }^{10} 0,29 \mathrm{~m} / \mathrm{s} \mathrm{e} \mathrm{2,88 \%}$ respectivamente, com os resultados da presente pesquisa (amostra A, velocidade de fluidização de $0,465 \mathrm{~m} / \mathrm{s}$ e carbono residual de $2,41 \%$ ) é possível afirmar sobre o bom desempenho do processo de combustão de casca de arroz em leito fluidizado para a obtenção de cinza com alto teor de sílica e baixa perda ao fogo.

\section{Composição de fases da CCA}

Um típico difratograma de raios $\mathrm{X}$ é apresentado na Figura 2, correspondente ao teste A. As amostras de cinza obtidas a partir da combustão da CA em leito fluidizado borbulhante e atmosférico mantiveram-se predominantemente amorfas para todas as condições experimentais propostas. Os difratogramas apresentaram um halo de baixa intensidade entre 15 e $30^{\circ}$, de acordo com relatos encontrados na literatura. ${ }^{19,20} \mathrm{Os}$ picos associados à presença de espécies cristalinas são atribuídos a contaminações do material inerte utilizado, areia (sílica cristalina na forma de quartzo), necessário para desenvolver o processo de fluidização da casca de arroz. Armesto et al. ${ }^{8}$ e Rozainee et al. ${ }^{10}$ também atribuíram a presença de cristais de quartzo, na cinza, ao arraste da areia para fora do reator na combustão de CA de arroz em leito fluidizado borbulhante e atmosférico.

A areia utilizada (tamanho médio de $242 \mu \mathrm{m}$ ) tem velocidade mínima de fluidização em torno de $0,12 \mathrm{~m} / \mathrm{s}$, em comparação com os valores de 0,33 e $0,43 \mathrm{~m} / \mathrm{s}$ de sua mistura com casca de arroz em proporções mássicas de até $7,1 \%$. Assim, a possibilidade de que exista elutriação das partículas do material inerte para fora do reator é considerável.

\section{Distribuição de tamanho de partículas da CCA}

A Figura 3 mostra a distribuição de tamanho de partículas (DTP) via difração a laser para as amostras de cinza obtidas após a combustão de CA. Os resultados correspondem, de modo geral, a um comportamento gaussiano, com tamanhos médios entre 176 e $352 \mu \mathrm{m}$. Para as amostras C e D, evidencia-se uma natureza bimodal, que confirma a presença de areia (material inerte do leito), igualmente identificada nos resultados de DRX. 


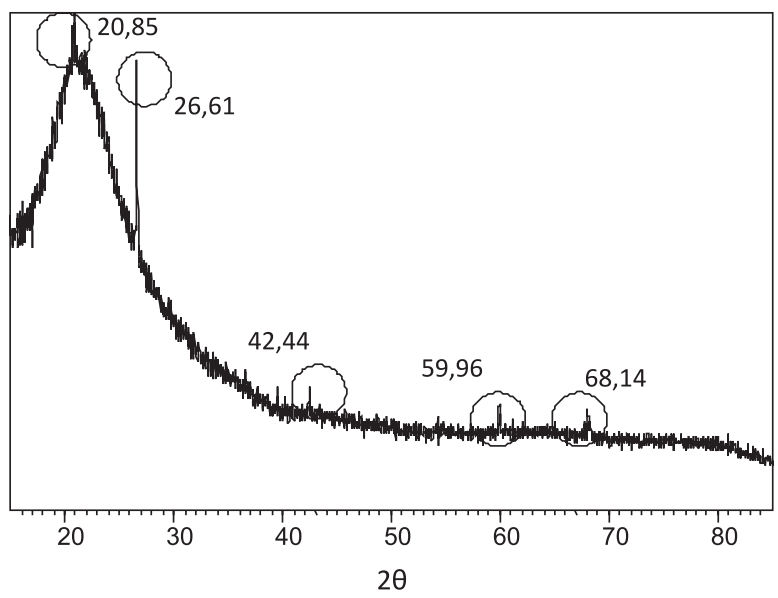

Figura 2. Difratograma de raios $X$ da amostra $A$ de $C C A$

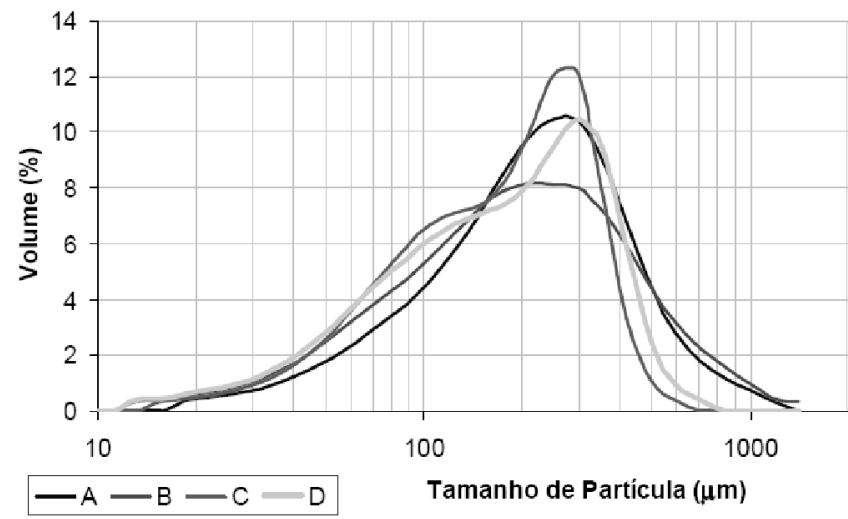

Figura 3. Distribuição de tamanho de partículas da CCA

Os resultados de tamanho médio de partículas, obtidos por peneiramento, são apresentados na Tabela 5. As diferenças entre os valores de tamanho médio são atribuídas às características intrínsecas de cada método. Em especial, a DTP por difração a laser ocorre em meio aquoso, que pode propiciar formação de aglomerados, assim como erros na detecção do perfil geométrico da partícula, pois o método assume que a forma da partícula é uma esfera.

Tabela 5. Tamanho médio de partícula por peneiramento

\begin{tabular}{lc}
\hline Amostra & Tamanho médio $(\mu \mathrm{m})$ \\
\hline A & 101 \\
B & 88 \\
C & 99 \\
D & 91 \\
\hline
\end{tabular}

Valores de tamanho médio de partículas de CCA obtidas a partir da combustão de CA em leito fluidizado relatados na literatura oscilam entre 45 e $108 \mu \mathrm{m} .{ }^{9,10}$

\section{Microestrutura de partículas da CCA}

As micrografias obtidas por MEV das partículas de CCA permitiram identificar uma aparência de espiga de milho com menores aumentos (30×, Figura 4a). Com aumento intermediário (200×, Figura 4b), é possível apreciar uma estrutura lamelar e porosa na (a)

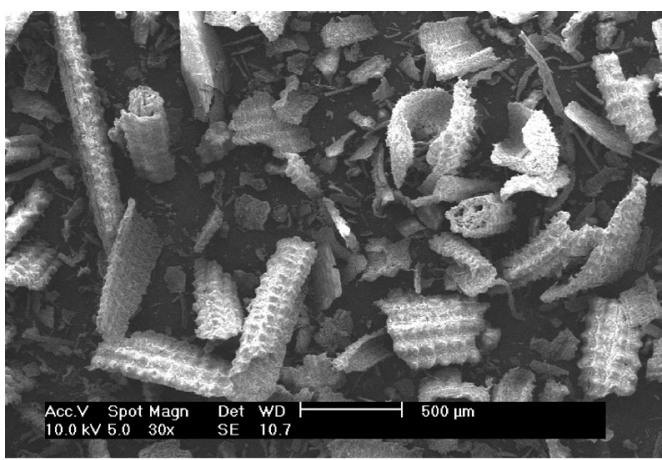

(b)

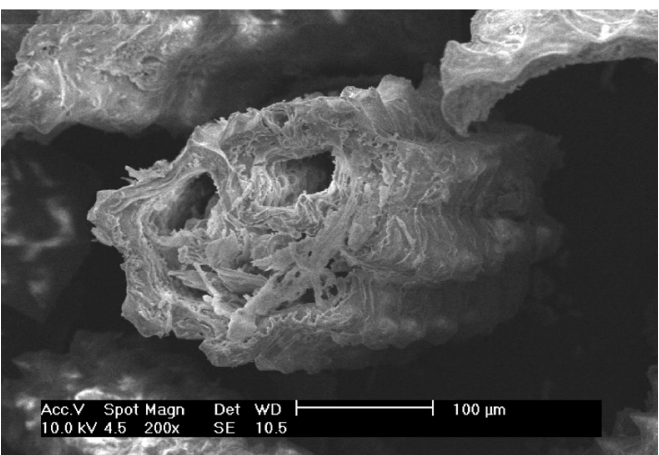

(c)

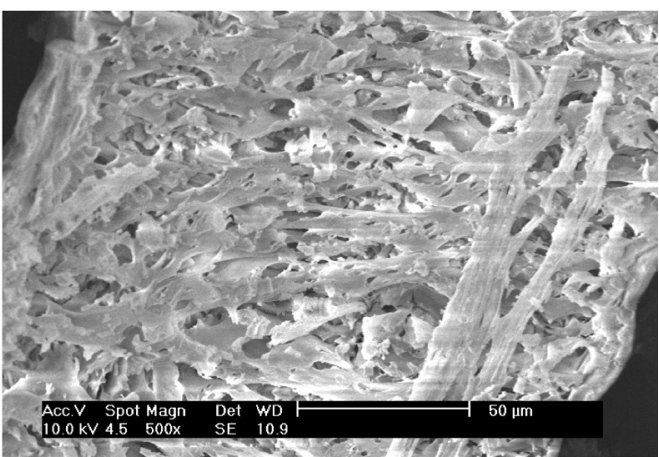

Figura 4. Micrografias (MEV) de amostras de CCA com diferentes aumentos: (a) $30 \times$, , b) $200 \times$, (c) $500 \times$

parte interna da casca, atribuída à oxidação dos compostos orgânicos, que foram convertidos em gases quentes durante o processo de combustão. Com maiores aumentos (500×, Figura 4c), observa-se um material altamente poroso correspondente à sílica com considerável área superficial, como relatado na literatura. ${ }^{21}$

Finalmente, as partículas de CCA tendem a manter o formato original após a transformação termoquímica em leito fixo, embora sejam quebradiças e facilmente convertidas em pó muito fino mediante moagem..$^{22}$ Para o caso dos leitos fluidizados, em que existem turbulências e convecções elevadas no interior do reator, a morfologia da cinza mostra semelhanças ao seu estado in natura (ondulações na epiderme externa), como observado anteriormente por outros pesquisadores. $^{23}$

\section{CONCLUSÕES}

Os resultados da presente pesquisa demonstram a viabilidade técnica de valorizar um resíduo como a casca de arroz, para a obtenção de cinza com altos teores de sílica amorfa (>93\%) e baixos teores de carbono (perda ao fogo inferior a 3\%), a partir da transformação termoquímica num reator de leito fluidizado borbulhante e atmosférico. 
Foi evidenciado o potencial desse tipo de reator com relação à rapidez, continuidade e auto-suficiência energética do processo, comparativamente a leitos fixos, que empregam fontes externas de energia e longos tempos de reação para obtenção da sílica amorfa.

Os difratogramas de raios $\mathrm{X}$ das amostras de cinza obtidas apresentaram fases amorfas, caracterizadas por um halo de relativa baixa intensidade entre 15 e $30^{\circ}(2 \theta)$. Os picos associados à presença de espécies cristalinas (quartzo) são atribuídos a contaminações pelo material inerte utilizado (areia), produto do arraste dessas para fora do reator. Os resultados de distribuição de tamanho de partícula (DTP) pelo método laser, particularmente para as amostras C e E, mostraram a natureza bimodal que corrobora a existência de um material diferente na cinza.

A morfologia da CCA obtida em reator de leito fluidizado tende a se manter inalterada, mesmo após a transformação termoquímica, com resultados comparáveis aos obtidos por vários pesquisadores em conversões termoquímicas em leito fixo.

\section{MATERIAL SUPLEMENTAR}

Está disponível em http://quimicanova.sbq.org.br, na forma de arquivo pdf, com acesso livre.

\section{AGRADECIMENTOS}

Às empresas Andercol S.A., Premac S.A. e Tinpes S.A. (Colômbia) pelo suporte no desenvolvimento da pesquisa e ao Instituto Colombiano para o Desenvolvimento da Ciência e Tecnologia pelo auxílio financeiro.

\section{REFERÊNCIAS}

1. De Souza, M. F.; Magalhães, W. L. E.; Persegil, M. C.; Mater. Res. 2002, $5,467$.

2. Krishnarao, R. V.; Subrahmanyam, J.; Jagadish Kumar, T.; J. Eur. Ceram. Soc. 2001, 21, 99.

3. Chandrasekhar, S.; Pramada, P. N.; Praveen, L.; J. Mater. Sci. 2003, 38, 3159 .
4. Chandrasekhar, S.; Pramada, P. N.; Praveen, L.; J. Mater. Sci. 2005, 40, 6535.

5. Yalçin, N.; Sevinç, V;. Ceram. Int. 2001, 27, 219.

6. Della, V. P.; Junkes, J. A.; Oliveira, A. P. N.; Hotza, D.; Quim. Nova 2006, 29, 1175.

7. Junkes, J. A.; Della, V. P.; Acchar, W.; Oliveira, A. P. N.; Hotza, D.; Ind. Ceram. 2006, 26, 11.

8. Armesto, L.; Bahillo, A.; Veijonen, K.; Cabanillas, A.; Otero, J.; Biomass \& Bioenergy 2002, 23, 171.

9. Ramírez, J. J.; Martínez, J. D.; Monografías y Tesis 2007, 4, 186.

10. Rozainee, M.; Ngo, S. P.; Salema, A. A.; Tan, K. G.; Ariffin, M.; Zainura, Z. N.; Bioresour. Technol. 2007, 99, 703.

11. Rao, T. R.; Bheemarasetti, J. V. R.; Energy 2001, 26, 633.

12. Fang, M.; Yang, L.; Chen, G.; Shi, Z.; Luo, Z.; Cen, K.; Fuel Process. Technol. 2004, 85, 1273.

13. Qiaoqun, S.; Huilin, L.; Wentie, L.; Yurong, H.; Lidan, Y.; Gidaspow, D.; Fuel 2005, 84, 1739.

14. Huang, S.; Jing, S.; Wang, J.; Wang, Z.; Jin, Y.; Powder Technol. 2001, 117, 232.

15. Associação Brasileira de Normas Técnicas; NBR 9644: Preparação de amostras para análise química de materiais refratários, ABNT, 1986.

16. Associação Brasileira de Normas Técnicas; NBR 12860: Materiais refratários magnesianos - Análise química por espectrometria de fluorescência de raios-X, ABNT, 1993.

17. American Society for Testing and Materials; ASTM E11 - 04 Standard Specification for Wire Cloth and Sieves for Testing Purposes, ASTM, 2004.

18. Fernandes, M.; Tese de Doutorado, Universidade Estadual de Campinas, Brasil, 2004

19. Hamdan, H.; Mohd Muhid, M. N.; Endud, S.; Listiorini, E.; Ramli, Z. $\mathrm{Si}$; J. Non-Cryst. Solids 1997, 211, 126.

20. Jauberthiea, R.; Rendell, F.; Tamba, S.; Cisse, I.; Constr. \& Build. Mater. 2000, $14,419$.

21. Liou, T-H.; Mater. Sci. Eng., A 2004, 364, 313.

22. Della, V. P.; Tese de Doutorado, Universidade Federal de Santa Catarina, Brasil, 2005.

23. Skrifvars, B-J.; Yrjas, P.; Laurén, T.; Kinni, J.; Tran, H.; Hupa, M.; Energy Fuels 2005, 19, 1512. 


\section{CARACTERIZAÇÃO DE CINZA OBTIDA POR COMBUSTÃO DE CASCA DE ARROZ EM REATOR DE LEITO} FLUIDIZADO

Juan Daniel Martínez Angel e Tatiana Gisset Pineda Vásquez

Grupo de Investigaciones Ambientales, Universidad Pontifícia Bolivariana, Medellín, Colômbia Janaina Accordi Junkes e Dachamir Hotza*

Departamento de Engenharia Química e Engenharia de Alimentos, Universidade Federal de Santa Catarina, $88040-900$ Florianópolis - SC, Brasil

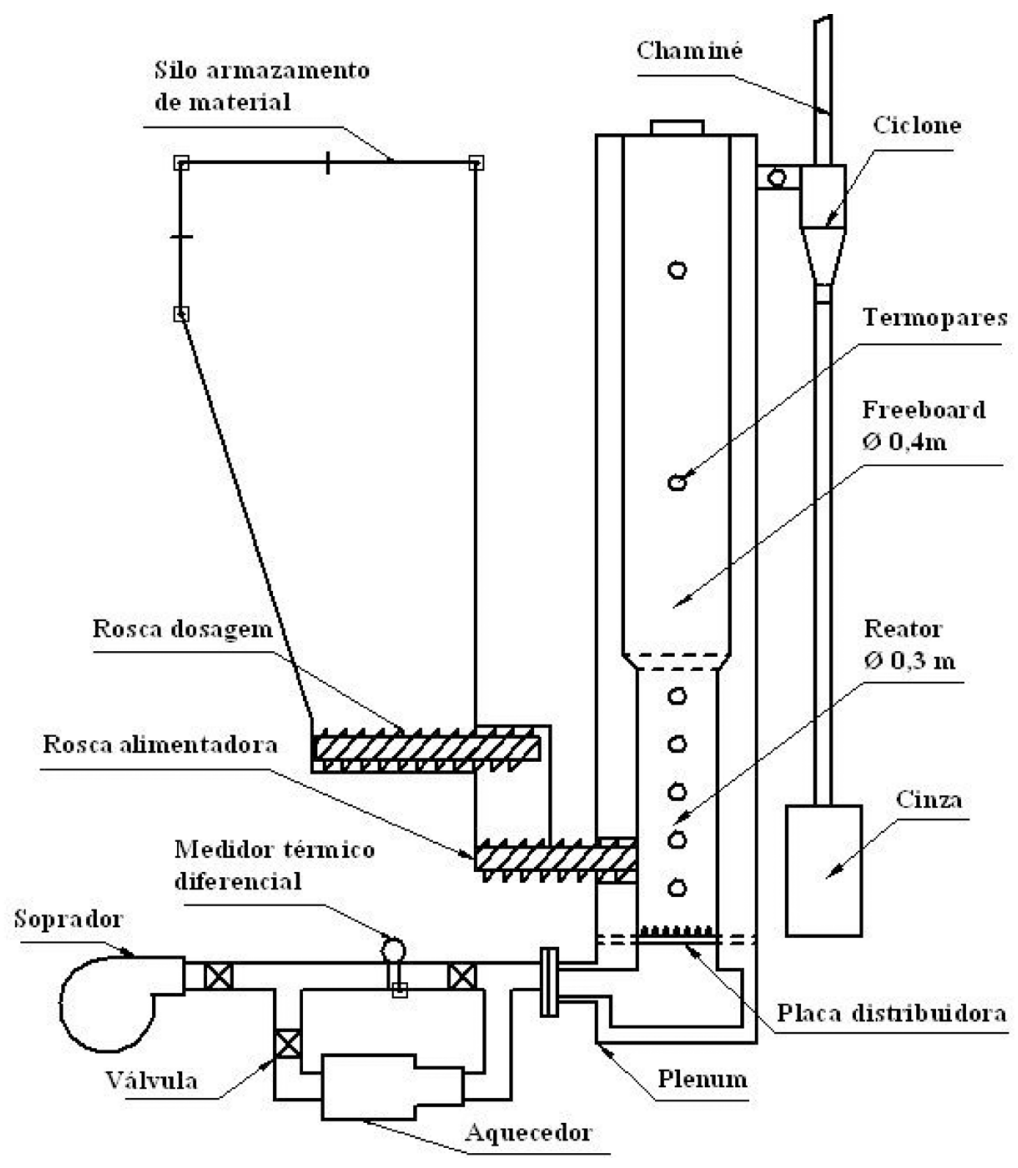

Figura 1S. Esquema do reator de leito fluidizado

O reator de leito fluidizado é mostrado esquematicamente na Figura 1S.

Os difratogramas de raios X correspondentes ao testes B, C e D são apresentados na Figura 2S. As amostras de cinza são, tal como para o teste $\mathrm{A}$, predominantemente amorfas, sendo os picos cristalinos associados à fase sílica devido à contaminação por areia. 


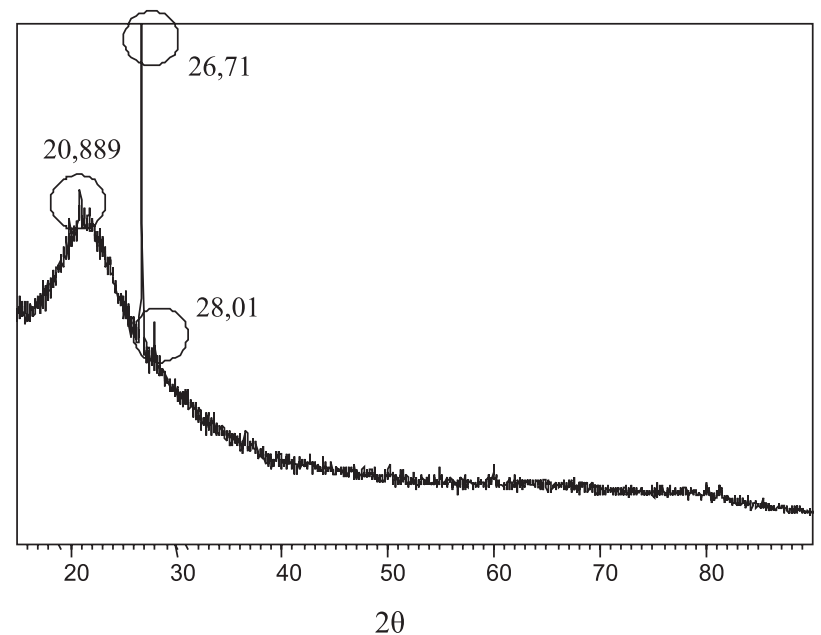

(a)

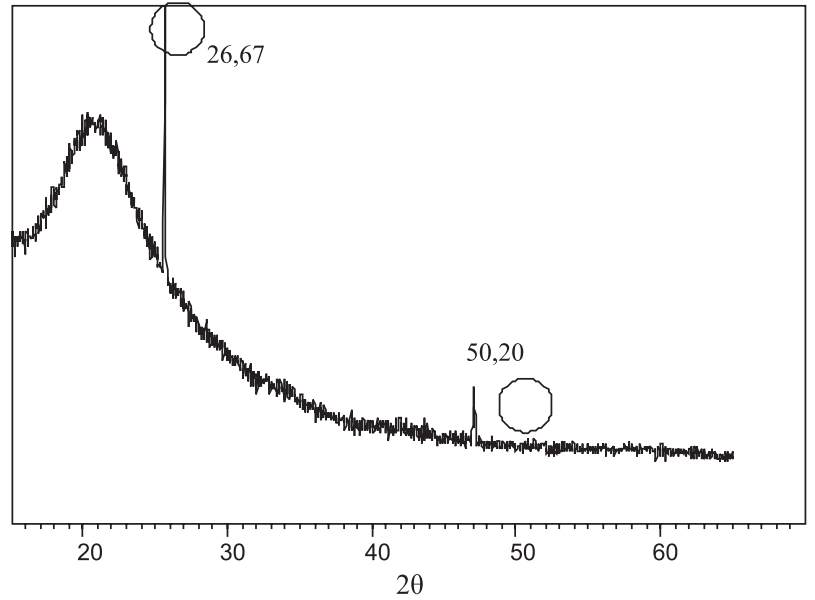

(b)

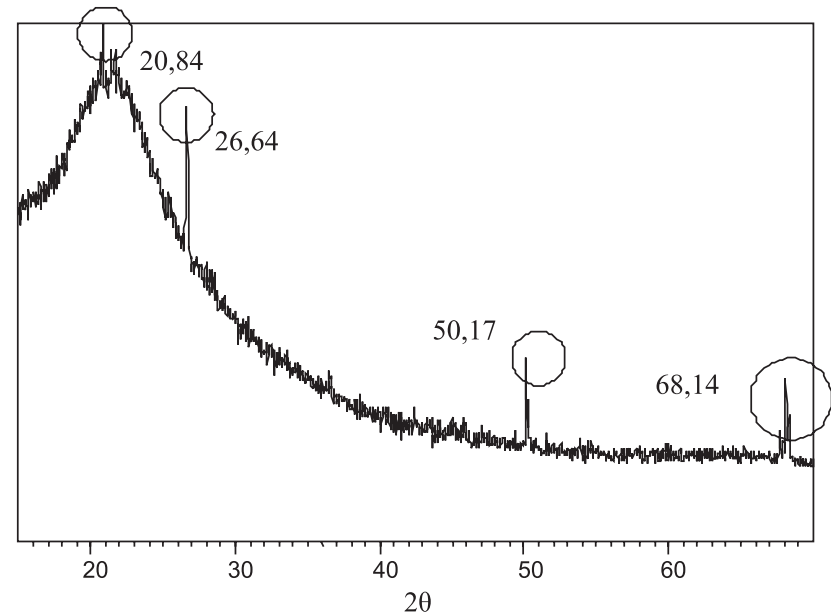

(c)

Figura 2S. Difratogramas de raios $X$ das amostras de CCA: (a) teste $B,(b)$ teste $C$, (c) teste $D$ 

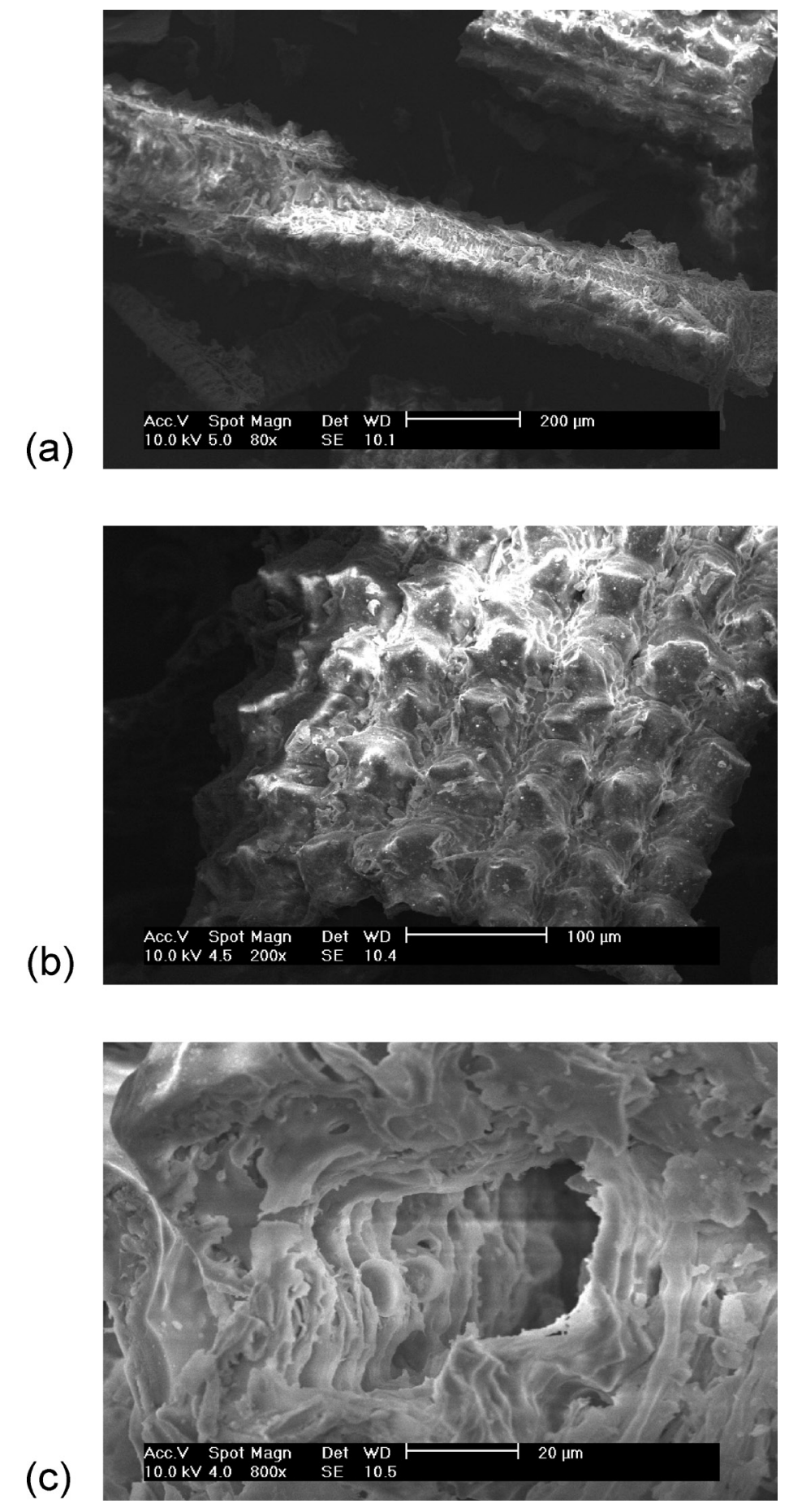

Figura 3S. Micrografias (MEV) de amostras de CCA com diferentes aumentos: (a) 80×, (b) 200×, (c) 800×

As micrografias da Figura 3S (a,b,c) mostram uma aparência de espiga de milho, uma estrutura lamelar e uma alta porosidade para diferentes aumentos (respectivamente $80 \times, 200 \times, 800 \times$ ). 